\title{
ERRATUM
}

\section{Factorial Validity of the Perceived Empathic and Social Self-Efficacy Scale for Adults with Serious Mental Illness: A Brief Report - ERRATUM}

Jennifer Sánchez, Joseph S. Pfaller, Kanako Iwanaga, Fong Chan, Connie Sung, Jill L. Bezyak and Shu-Mei Chen

DOI: https://doi.org/10.1017/jrc.2016.10, Published by Cambridge University Press, 13 December 2016

Keywords: empathy, social functioning, serious mental illness, confirmatory factor analysis

The order of the authors were listed incorrectly in the above publication (Jennifer Sánchez et al., 2016). The correct authorship is provided below:

Jennifer Sánchez ${ }^{1}$, Joseph S. Pfaller ${ }^{2}$, Connie Sung ${ }^{3}$, Jill L. Bezyak ${ }^{4}$, Kanako Iwanaga $^{5}$, Shu-Mei Chen ${ }^{6}$, and Fong Chan ${ }^{7}$

${ }^{1}$ Department of Rehabilitation and Counselor Education, The University of Iowa, Iowa City, IA, USA

${ }^{2}$ Department of Rehabilitation Psychology and Special Education, University of Wisconsin-Madison, Madison, WI, USA

${ }^{3}$ Department of Counselling, Educational Psychology and Special Education, Michigan State University, East Lansing, MI, USA

${ }^{4}$ Rehabilitation Counselling and Sciences, University of Northern Colorado, Greeley, CO, USA

${ }^{5}$ Department of Rehabilitation Psychology and Special Education, University of Wisconsin-Madison, Madison, WI, USA

${ }^{6}$ Tsaotun Psychiatric Center, Tsaotun, Taiwan

${ }^{7}$ Department of Rehabilitation Psychology and Special Education, University of Wisconsin-Madison, Madison, WI, USA

The authors and Cambridge University Press apologise to readers for these errors.

\section{Reference}

Sánchez, J., Pfaller, J., Iwanaga, K., Chan, F., Sung, C., Bezyak, J., \& Chen, S. (2016). Factorial Validity of the Perceived Empathic and Social Self-Efficacy Scale for Adults with Serious Mental Illness: A Brief Report. The Australian Journal of Rehabilitation Counselling, 22(2), 135-146. doi:10.1017/jrc.2016.10 